\title{
Robust Medical Images Segmentation Using Learned Shape and Appearance Models
}

\author{
Ayman El-Baz ${ }^{1}$ and Georgy Gimel'farb ${ }^{2}$ \\ 1 Bioimaging Laboratory, Bioengineering Department, University of Louisville, \\ Louisville, KY, USA \\ 2 Department of Computer Science, University of Auckland, Auckland, New Zealand
}

\begin{abstract}
We propose a novel parametric deformable model controlled by shape and visual appearance priors learned from a training subset of co-aligned medical images of goal objects. The shape prior is derived from a linear combination of vectors of distances between the training boundaries and their common centroid. The appearance prior considers gray levels within each training boundary as a sample of a Markov-Gibbs random field with pairwise interaction. Spatially homogeneous interaction geometry and Gibbs potentials are analytically estimated from the training data. To accurately separate a goal object from an arbitrary background, empirical marginal gray level distributions inside and outside of the boundary are modeled with adaptive linear combinations of discrete Gaussians (LCDG). Due to the analytical shape and appearance priors and a simple Expectation-Maximization procedure for getting the object and background LCDG, our segmentation is considerably faster than with most of the known geometric and parametric models. Experiments with various goal images confirm the robustness, accuracy, and speed of our approach.
\end{abstract}

\section{Introduction}

Parametric and geometric deformable models are widely used for image segmentation. However, in many applications, especially in medical image analysis, accurate segmentation with these models is a challenging problem due to noisy or low-contrast 2D/3D images with fuzzy boundaries between goal objects (e.g., anatomical structures) and their background; the similarly shaped objects with different visual appearances, and discontinuous boundaries because of occlusions or the similar visual appearance of adjacent parts of objects of different shapes [12]. Prior knowledge about the goal shape and/or visual appearance helps in solving such segmentation problems [2].

Relationship to the Prior Works. Initial attempts to involve the prior shape knowledge were built upon the edges. Pentland and Sclaroff [3] described an evolving curve with shape and pose parameters of a parametric set of points matched to strong image gradients and use a linear combination of eigenvectors to represent variations from an average shape. A parametric point model of Staib and Duncan [4] was based on an elliptic Fourier decomposition of landmarks. 
Model parameters ensure the best match between the evolving curve and points of strong gradients.

More efficient results were obtained by learning the priors from a training set of manually segmented images of goal objects. Pizer et al. 5] and Styner et al. 6] segment 3D medical images by coarse-to-fine deformation of a shapebased medial representation ("m-rep"). A deformable model of Huang et al. [7] integrates region, shape and interior signal features assuming an approximate region shape is a priori known and aligned with the image to initialize the model. Leventon et al. [8] and Shen et al. [9] augment a level set-based energy function guiding the evolution with special terms attracting to more likely shapes specified with the principal component analysis (PCA) of the training set of goal objects, while Chen et al. [10 use a geometric model with the prior "average shape". The most advanced level set-based geometric model of Tsai et al. 1] evolves as zero level of a $2 \mathrm{D}$ map of the signed shortest distances between each pixel and the boundary. The goal shapes are approximated with a linear combination of the training distance maps for a set of mutually aligned training images. High dimensionality of the distance map space hinders PCA, and to simplify the model, only a few top-rank principal components are included to the linear combination 11 .

Our approach follows the same ideas of using both the shape and appearance prior knowledge [11312, but differs in the three aspects. First, instead of using the level set framework with linear combinations of the distance maps or statistical classifier (e.g., support vector machine), we use a simple parametric deformable model. Both the model and each goal shape are represented by piecewise-linear boundaries with a predefined number of control points. Corresponding points are positioned on roughly equiangular rays from the common center being the centroid of the control points along each boundary. A robust wave propagation is used to find correspondences in an aligned pair of the boundaries. Secondly, visual appearance of the goal objects is roughly described by characteristic statistics of gray level co-occurrences. Grayscale object pattern is considered as a sample of a spatially homogeneous Markov-Gibbs random field (MGRF) with multiple pairwise interaction. The interaction parameters of MGRF are estimated analytically. Third, the evolution is also guided at each step with a first-order probability model of the current appearance of a goal object and its background.

\section{Basic Notation.}

○ $(x, y)$ - Cartesian coordinates of

$\circ \mathbf{R}=[(x, y): x=0, \ldots, X-1 ; y=0, \ldots, Y-1]$ - a finite arithmetic lattice supporting digital images and their region maps.

$\circ \mathbf{g}=\left[g_{x, y}:(x, y) \in \mathbf{R} ; g_{x, y} \in \mathcal{Q}\right]$ - a grayscale digital image taking values from a finite set $\mathcal{Q}=\{0, \ldots, Q-1\}$.

$\circ \mathbf{m}=\left[m_{x, y}:(x, y) \in \mathbf{R} ; m_{x, y} \in \mathbf{L}\right]$ - a region map taking labels from a binary set $\mathbf{L}=\{\mathrm{ob}, \mathrm{bg}\}$; each label $m_{x, y}$ indicates whether the pixel $(x, y)$ in the corresponding image $\mathbf{g}$ belongs to a goal object (ob) or background (bg). 
$\circ \mathbf{b}=\left[\mathbf{p}_{k}: k=1, \ldots, K\right]-$ a deformable piecewise-linear boundary with the $K$ control points $\mathbf{p}_{k}=\left(x_{k}, y_{k}\right)$ forming a circularly connected chain of line segments $\left(\mathbf{p}_{1}, \mathbf{p}_{2}\right), \ldots,\left(\mathbf{p}_{K-1}, \mathbf{p}_{K}\right),\left(\mathbf{p}_{K}, \mathbf{p}_{1}\right)$.

$\circ \mathbf{d}=\left[d_{k}^{2}: k=1, \ldots, K\right]-$ a vector description of the boundary $\mathbf{b}$ in terms of the square distances $d_{k}^{2}=\left(x_{k}-x_{0}\right)^{2}+\left(y_{k}-y_{0}\right)^{2}$ from the control points to the model centroid $\mathbf{p}_{0}=\left(x_{0}=\frac{1}{K} \sum_{k=1}^{K} x_{k}, y_{0}=\frac{1}{K} \sum_{k=1}^{K} y_{k}\right)$, i.e. to the point at the minimum mean square distance from all the control points.

$\circ \mathbf{S}=\left\{\left(\mathbf{g}_{t}, \mathbf{m}_{t}, \mathbf{b}_{t}, \mathbf{d}_{t}\right): t=1, \ldots, T\right\}-$ a training set of grayscale images of the goal objects with manually prepared region maps and boundary models.

$\circ|\mathbf{A}|$ - the cardinality of a finite set $\mathbf{A}$.

\section{Shape Prior}

To build the shape prior, all the training objects in $\mathbf{S}$ are mutually aligned to have the same centroid and unified poses (orientations and scales of the objects boundaries) as in Fig. 1(a). For the definiteness, let each training boundary $\mathbf{b}_{t} \in$ $\mathbf{S}$ is represented with $K$ control points on the polar system of $K^{\circ}$ equiangular rays (i.e. with the angular pitch $2 \pi / K^{\circ}$ ) emitted from the common centroid $\mathbf{p}_{0}$. The rays are enumerated clockwise, with zero angle for the first position $\mathbf{p}_{t, 1}$ of each boundary. Generally, there may be rays with no or more than one intersection of a particular boundary, so that the number of the control points $K$ may differ from the number of the rays $K^{\circ}$. Because the training boundaries $\mathbf{b}_{t} \in \mathbf{S} ; t=1, \ldots, T$, share the same centroid $\mathbf{p}_{0}$, any linear combination $\mathbf{d}=$ $\sum_{t=1}^{T} w_{t} \mathbf{d}_{t}$ of the training distance vectors defines a unique new boundary $\mathbf{b}$ with the same centroid. Typically, shapes of the training objects are very similar, and their linear combinations could be simplified by the PCA to escape singularities when adjusting to a given boundary.

Let $\mathbf{D}=\left[\begin{array}{llll}\mathbf{d}_{1} & \mathbf{d}_{2} & \cdots & \mathbf{d}_{T}\end{array}\right]$ and $\mathbf{U}=\mathbf{D D}^{\top}$ denote the $K \times T$ matrix with the training distance vectors as columns and the symmetric $K \times K$ Gram matrix of sums of squares and pair products $\sum_{t=1}^{T} d_{t, k} d_{t, k^{\prime}} ; k, k^{\prime}=1, \ldots, K$ of their components, respectively. The PCA of the matrix $\mathbf{U}$ produces $K$ eigen-vectors $\left[\mathbf{e}_{i}: i=1, \ldots, K\right]$ sorted by their eigenvalues $\lambda_{1} \geq \lambda_{2} \geq \ldots \geq \lambda_{K} \geq 0$. Due to identical or very similar training shapes, most of the bottom-rank eigenvalues are zero or very small, so that the corresponding "noise" eigenvectors can be discarded. Only a few top-rank eigenvectors actually represent the training shapes; the top distance eigenvector $\mathbf{e}_{1}$ corresponds to an "average" shape and a few others determine its basic variability. For simplicity, we select the toprank subset of the eigenvectors $\left(\mathbf{e}_{i}: i=1, \ldots, K^{\prime}\right) ; K^{\prime}<K$ by thresholding: $\sum_{i=1}^{K^{\prime}} \lambda_{i} \approx \theta \sum_{i=1}^{K} \lambda_{i}$ with an empirical threshold $\theta=0.8 \ldots 0.9$.

An arbitrary boundary $\mathbf{b}_{\mathrm{c}}$ aligned with the training set is described with the vector $\mathbf{d}_{\mathrm{c}}$ of the squared distances from its control points to the centroid. The prior shape approximating this boundary is specified by the linear combination of the training vectors: $\mathbf{d}^{*}=\sum_{i=1}^{K^{\prime}} w_{i}^{*} \mathbf{e}_{i} \equiv \sum_{i=1}^{K^{\prime}}\left(\mathbf{e}_{i}^{\top} \mathbf{d}_{\mathrm{c}}\right) \mathbf{e}_{i}$. Each signed difference 


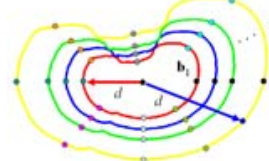

(a)

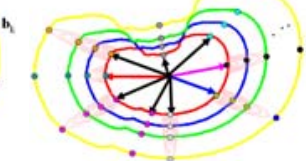

(b)

Fig. 1. (a) Mutually aligned training boundaries and (b) searching for corresponding points between the mutually aligned training boundaries

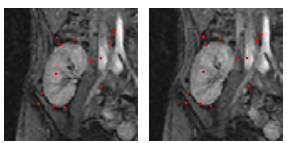

$(\mathrm{a}-\mathrm{b})$

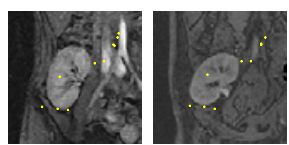

$(\mathrm{a}-\mathrm{c})$
Fig. 2. Corresponding points found by SIFT in each pair $(a, b)$, and $(a, c)$ shown in red and yellow, respectively

$\Delta_{k}=d_{k}^{*}-d_{\mathrm{c}, k}$ determines the direction and force to move the boundary $\mathbf{b}_{\mathrm{c}}$ towards the closest shape prior $\mathbf{b}^{*}$ specified by the distance vector $\mathbf{d}^{*}$.

Search for Corresponding Points is performed to suppress local "noise" (spurious deviations) in the training boundaries. The corresponding points are found in the aligned training boundaries by a robust wave-propagation based search (see Fig. 1(b)). An orthogonal wave is emitted from a point in one boundary, and the point at which the maximum curvature position of the wave front hits the second boundary is considered as the corresponding point.

SIFT-based Alignment. Just as the conventional level-set based geometric models with the shape priors, e.g. in [1], our approach depends essentially on accuracy of mutual alignment of similar shapes at both the training and segmentation stages. In the latter case the deformable model is initialized by aligning an image $\mathbf{g}$ to be segmented with one of the training images, say, $\mathbf{g}_{1} \in \mathbf{S}$, arbitrarily chosen as a prototype.

First we use the scale invariant feature transform (SIFT) proposed by Lowe [14] to reliably determine a number of point-wise correspondences between two images under their relative affine geometric and local contrast / offset signal distortions. Then the affine transform aligning $\mathbf{g}$ most closely to $\mathbf{g}_{1}$ is determined by the gradient descent minimization of the mean squared positional error between the corresponding points.

Figure 2 shows correspondences found by SIFT in Dynamic Contrast Enhanced Resonance Imaging (DCE-MRI) images of human kidneys. The resulting affinely aligned goal shapes have roughly the same center and similar poses (orientations and scales). Quality of such alignment is evaluated in Fig. 3] by
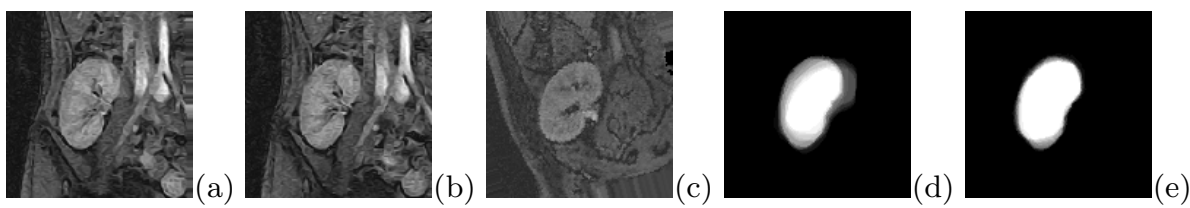

Fig. 3. Mutually aligned images shown in Fig. 2 (a, b, c), and overlaps of the training region maps before $(\mathrm{d})$ and after (e) the alignment 
averaging all the training region maps $\mathbf{m}_{t} ; t=1, \ldots, T$, before and after the training set $\mathbf{S}$ is mutually aligned. It is clear that the alignment step reduced the variations between the co-aligned objects, SIFT based registration approach results in more accurate average shape model that is useful e.g. in any shape based segmentation framework.

\section{Appearance Models}

MGRF-based Appearance Prior. Our appearance prior is a rough descriptor of typically complex grayscale patterns of goal objects in terms of only secondorder signal statistics. Each goal image is considered as a sample of a pairwise Markov-Gibbs random field (MGRF).

Let $\mathcal{N}=\left\{\left(\xi_{i}, \eta_{i}\right): i=1, \ldots, n\right\}$ be a finite set of $(x, y)$-offsets specifying neighbors $\{((x+\xi, y+\eta),(x-\xi, y-\eta)):(\xi, \eta) \in \mathcal{N}\} \wedge \mathbf{R}$ interacting with each pixel $(x, y) \in \mathbf{R}$. Let $\mathcal{C}_{\xi, \eta}$ be a family of pairs $c_{\xi, \eta ; x, y}=((x, y),(x+\xi, y+\eta))$ in $\mathbf{R}$ with the offset $(\xi, \eta) \in \mathcal{N}$, i.e. the family of translation invariant pairwise cliques of the neighborhood graph on $\mathbf{R}$. Let $\mathbf{V}$ be a vector of Gibbs potentials for gray level co-occurrences in the neighboring pairs: $\mathbf{V}^{\top}=\left[\mathbf{V}_{\xi, \eta}^{\top}:(\xi, \eta) \in \mathcal{N}\right]$ where $\mathbf{V}_{\xi, \eta}^{\top}=\left[V_{\xi, \eta}\left(q, q^{\prime}\right):\left(q, q^{\prime}\right) \in \mathcal{Q}^{2}\right]$. A generic MGRF with multiple pairwise interaction on $\mathbf{R}$ is specified by the Gibbs probability distribution (GPD)

$$
P(\mathbf{g})=\frac{1}{Z} \exp \sum_{(\xi, \eta) \in \mathcal{N}} \sum_{c_{\xi, \eta ; x, y} \in \mathcal{C}_{\xi, \eta}} V_{\xi, \eta}\left(g_{x, y}, g_{x+\xi, y+\eta}\right) \equiv \frac{1}{Z} \exp |\mathbf{R}| \mathbf{V}^{\top} \mathbf{F}(\mathbf{g})
$$

Here, $Z$ is the partition function, $\mathbf{F}^{\mathbf{\top}}(\mathbf{g})$ is the vector of scaled empirical probability distributions of gray level co-occurrences over each clique family: $\mathbf{F}^{\top}(\mathbf{g})=$ $\left[\rho_{\xi, \eta} \mathbf{F}_{\xi, \eta}^{\top}(\mathbf{g}):(\xi, \eta) \in \mathcal{N}\right]$ where $\mathbf{F}_{\xi, \eta}(\mathbf{g})=\left[f_{\xi, \eta}\left(q, q^{\prime} \mid \mathbf{g}\right):\left(q, q^{\prime}\right) \in \mathcal{Q}^{2}\right]^{\top}$ and $\rho_{\xi, \eta}=\frac{\left|\mathcal{C}_{\xi, \eta}\right|}{|\mathbf{R}|}$ is the relative size of the clique family.

To identify the MGRF model described in Eq. (11), we have to estimate the Gibbs Potentials V. In this paper we introduce a new analytical maximum likelihood estimation for the Gibbs potentials (the mathematical proof for this new estimator is shown on our website).

$$
V_{\mathrm{ob} ; \xi, \eta}\left(q, q^{\prime}\right)=\lambda\left(f_{\mathrm{ob} ; \xi, \eta}\left(q, q^{\prime}\right)-1 / Q^{2}\right)
$$

where $\lambda$ is the analytically computed common scaling factor: $\lambda \approx Q^{2}$ if $Q \gg 1$ and $\rho_{\text {ob } ; \xi, \eta} \approx 1$ for all $(\xi, \eta) \in \mathcal{N}$.

LCDG-models of Current Appearance. Non-linear intensity variations in a data acquisition system due to a scanner type and scanning parameters affect visual appearance of the object to be segmented. Thus, in addition to the learned appearance prior, we describe an on-going object appearance with a marginal intensity distribution within an evolving boundary $\mathbf{b}$ in $\mathbf{g}$. This distribution is considered as a dynamic mixture of two probability distributions that characterize the object and its background, respectively, and is partitioned into these two models using the EM-based approach in [15]. 


\section{Model Evolution}

The evolution $\mathbf{b}_{\tau} \rightarrow \mathbf{b}_{\tau+1}$ of the deformable boundary $\mathbf{b}$ in discrete time, $\tau=$ $0,1, \ldots$, is determined by solving the Eikonal equation $\left|\nabla T\left(\mathbf{p}_{k, \tau}\right)\right| F\left(\mathbf{p}_{k, \tau}\right)=$ $1 ; k=1, \ldots, K$, where $F(\mathbf{p})$ is a speed function for the control point $\mathbf{p}=[x, y]$ of the current boundary. Our speed function depends on the shape prior, the LCDG-model of current appearance, and the MGRF-based appearance prior:

$$
F(\mathbf{p})=e^{-\beta|\Delta|} p_{\text {pix }, \mathrm{ob}}\left(g_{x, y}\right) \pi_{x, y}\left(g_{x, y} \mid \mathbf{S}\right)
$$

Here, $\Delta$ is the signed distance between the current control point $\mathbf{p} \in \mathbf{b}_{\tau}$ and the like one in the closest shape prior along the ray from the current boundary centroid. The constant factor $\beta$ determines the evolution speed $(0<\beta<1$ for a smooth propagation). The marginal probabilities $p_{\text {pix ob }}(q)$ and $p_{\text {pix,bg }}(q)$ of the gray value $g_{x, y}=q$ are estimated with the LCDG-submodels for the object and its background, respectively. The prior conditional probability $\pi_{x, y}(q \mid \mathbf{S})$ of the gray value $g_{x, y}=q$ in the pixel $\mathbf{p}=(x, y)$, given the current gray values in its neighborhood, is estimated in line with the MGRF prior appearance model:

$$
\pi_{x, y}\left(g_{x, y} \mid \mathbf{S}\right)=\left(\exp \left(E_{x, y}\left(g_{x, y} \mid \mathbf{S}\right)\right)\right) /\left(\sum_{q \in \mathcal{Q}} \exp \left(E_{x, y}(q \mid \mathbf{S})\right)\right)
$$

where $E_{x, y}(q \mid \mathbf{S})$ is the pixel-wise Gibbs energy for the gray value $q$ in the pixel $\mathbf{p}=(x, y)$, given the fixed gray values in its characteristic neighborhood: $E_{x, y}(q \mid \mathbf{S})=$

$$
\sum_{(\xi, \eta) \in \mathbf{N}^{\prime}}\left(V_{\mathrm{ob} ; \xi, \eta}\left(g_{x-\xi, y-\eta}, q\right)+V_{\mathrm{ob} ; \xi, \eta}\left(q, g_{x+\xi, y+\eta}\right)\right) .
$$

\section{Experimental Results and Conclusions}

Performance of the proposed parametric deformable model was evaluated on a large number of dynamic contrast-enhanced MRI (DCE-MRI) of human kidneys with the ground truth presented by a radiologist. The DCE-MRI are usually noisy, with continuously changing and low contrast. Note that about $20 \%$ of images were used to learn the prior models for the kidney. Basic segmentation stages of the algorithm are shown in Fig. 4. A comparative results in Table 1 for 4000 kidney DCE-MR images confirm accuracy and robustness of our approach. It is clear from Table 1 that the statistical analysis using a two tailed t-test shows that there is a significant difference $\left(P<10^{-4}\right)$ between the error generated by our segmentation approach and the error generated by the algorithm in 1] or ASM algorithm in 16. Experiments with these DCE-MRI kidney images provide support for the proposed parametric deformable model guided with the learned shape and appearance priors. Our approach assumes that $(i)$ the boundaries of the training and test objects are reasonably similar to within a relative affine transform and (ii) SIFT reliably detects corresponding points to automatically align the goal objects in the images despite their different backgrounds. 
Table 1. Accuracy and time performance of our segmentation in comparison to Tsai et al.'s approach [1] and active shape model (ASM) [16]. One fifth of the kidney and corpus callosum images are used as training sets. Note that the training sets must contain all possible shape variability including pathology if it exists. Note that all these statistical results are calculated with respect to radiologist segmentation (ground truth).

\begin{tabular}{|l|c|c|c|c|c|c|}
\cline { 2 - 7 } \multicolumn{1}{c|}{} & \multicolumn{7}{c|}{ Seg. algorithm } \\
\cline { 2 - 7 } \multicolumn{1}{c|}{} & Our & {$[1$} & {$[16$} & Our & {$[1$} & {$[16$} \\
\hline Images(number) & \multicolumn{3}{|c|}{ Kidney $(4000)$} & \multicolumn{2}{c|}{ Corpus Callosum (200) } \\
\hline Minimum error, \% & $\mathbf{0 . 2 5}$ & 3.9 & 1.3 & $\mathbf{0 . 1 1}$ & 1.9 & 0.9 \\
Maximum error, \% & $\mathbf{1 . 5}$ & 8.3 & 10.6 & $\mathbf{0 . 4 7}$ & 4.1 & 4.9 \\
Mean error, \% & $\mathbf{0 . 8 3}$ & 5.8 & 5.95 & $\mathbf{0 . 2 7}$ & 3.1 & 3.71 \\
Standard deviation,\% & $\mathbf{0 . 4 5}$ & 1.5 & 3.7 & $\mathbf{0 . 2 1}$ & 0.97 & 1.17 \\
\hline Significant difference, P-value & & $<10^{-4}$ & $<10^{-4}$ & & $<10^{-4}$ & $<10^{-4}$ \\
Average time, sec & $\mathbf{2 3}$ & 253 & 7 & $\mathbf{1 1}$ & 153 & 3 \\
\hline
\end{tabular}
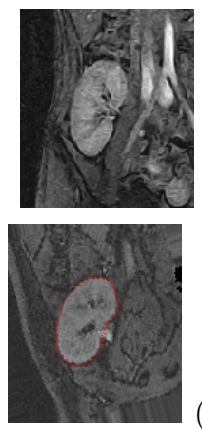

(a)

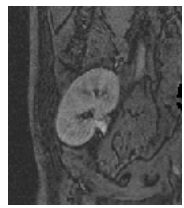

(e)

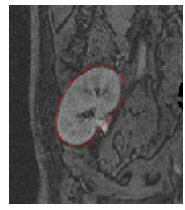

(b)
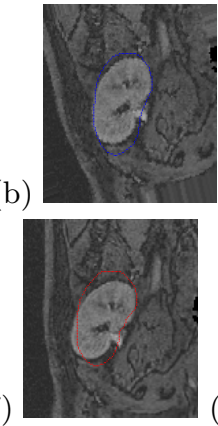

(c)

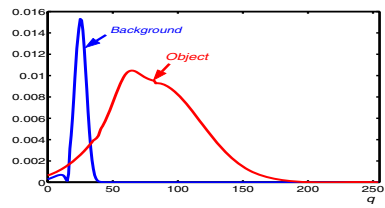

(d)

$(\mathrm{g})$

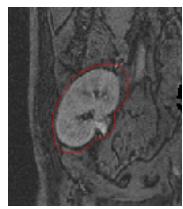

(h)

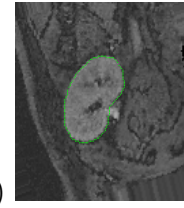

Fig. 4. Chosen training kidney prototype (a), an image to be segmented (b), its alignment to the prototype and initialization (in blue) (c), LCDG-estimates (d) of the marginal gray level distributions $\mathbf{p}_{\mathrm{pix}, \mathrm{ob}}$ and $\mathbf{p}_{\mathrm{pix}, \mathrm{bg}}$, final segmentation (in red) of the image aligned to the training prototype (e), the same result (f) after its inverse affine transform to the initial image (b) (the total error $0.63 \%$ comparing to the ground truth in green (i)), the segmentation (g) with the algorithm in [1] (the total error $4.9 \%$ comparing to the ground truth (i)), and the segmentation (h) with the algorithm in 16] (the total error $5.17 \%$ comparing to the ground truth (radiologist segmentation) (i)). Note that our results obtained using 140 points describing kidney shape.

Although these assumptions restrict an application area of our approach comparing to the conventional parametric models, the latter typically fail on the above and similar images. More accurate level set-based geometric models with linear combinations of the training distance maps as the shape priors also rely on the mutual image alignment. Compared to these models, our approach escapes some of theoretical inconsistencies, is computationally much simpler and faster, and has the similar accuracy on high-contrast images, but notably better performance on low-contrast and multimodal ones. Due to space limitations, similar results for more complex medical images such as corpus callosum (see Table 1), 
brain stem, and brain ventricles, are presented on our web site1. Also, we will post on our web site more results that demonstrate the effect of each model in the proposed segmentation framework. The proposed approach is suitable to segment pathological organs but a sample these pathological conditions must be included in the training data sets to be included in the prior shape model.

\section{References}

1. Tsai, A., Yezzi, A., Wells, W., Tempany, C., Tucker, D., Fan, A., Grimson, W., Willsky, A.: A shape based approach to the segmentation of medical imagery using level sets. IEEE TMI 22, 137-154 (2003)

2. Rousson, M., Paragios, N.: Shape priors for level set representations. In: Heyden, A., Sparr, G., Nielsen, M., Johansen, P. (eds.) ECCV 2002. LNCS, vol. 2351, pp. 78-92. Springer, Heidelberg (2002)

3. Pentland, A., Sclaroff, S.: Closed-form solutions for physically based shape modeling and recognition. IEEE PAMI 13, 715-729 (1991)

4. Staib, L., Duncan, J.: Boundary finding with parametrically deformable contour models. IEEE PAMI 14, 1061-1075 (1992)

5. Pizer, S., Gerig, G., Joshi, S., Aylward, S.: Multiscale medial shape-based analysis of image objects. Proc. IEEE 91, 1670-1679 (2003)

6. Styner, M., Gerig, G., Joshi, S., Pizer, S.: Automatic and robust computation of 3D medial models incorporating object variability. Int. J. Computer Vision 55, 107-122 (2002)

7. Huang, X., Metaxas, D., Chen, T.: MetaMorphs: Deformable shape and texture models. In: Proc. CVPR, vol. 1, pp. 496-503 (2004)

8. Leventon, M., Grimson, E., Faugeras, O.: Statistical shape influence in geodesic active contours. In: Proc. CVPR, pp. 316-323 (2000)

9. Shen, D., Davatzikos, C.: An adaptive-focus deformable model using statistical and geometric information. IEEE PAMI 22, 906-913 (2000)

10. Chen, Y., Thiruvenkadam, S., Tagare, H., Huang, F., Wilson, D., Geiser, E.: On the incorporation of shape priors into geometric active contours. In: IEEE Workshop Variational and Level Set Methods, pp. 145-152 (2001)

11. Pohl, K.M., Kikinis, R., Wells, W.M.: Active mean fields: Solving the mean field approximation in the level set framework. In: Karssemeijer, N., Lelieveldt, B. (eds.) IPMI 2007. LNCS, vol. 4584, pp. 26-37. Springer, Heidelberg (2007)

12. Song, Z., Awate, S.P., Licht, D.J., Gee, J.C.: Clinical neonatal brain MRI segmentation using adaptive nonparametric data models and intensity-based markov priors. In: Ayache, N., Ourselin, S., Maeder, A. (eds.) MICCAI 2007, Part I. LNCS, vol. 4791, pp. 883-890. Springer, Heidelberg (2007)

13. Awate, S., Tasdizen, T., Foster, N., Whitaker, R.: Adaptive markov modeling for mutual-information-based, unsupervised MRI brain-tissue classification. Medical Image Analysis 10(5), 726-739 (2006)

14. Lowe, D.G.: Distinctive image features from scale-invariant Keypoints. Int. J. Computer Vision 60, 91-110 (2004)

15. El-Baz, A., Gimel'farb, G.: EM Based Approximation of Empirical Distributions with Linear Combinations of Discrete Gaussians. In: ICIP 2007, San Antonio, Texas, USA, September 16-19, 2007, vol. IV, pp. 373-376 (2007)

16. Cootes, T., Taylor, C.: A mixture model for representing shape variation. In: Proc. of British Machine Vision, pp. 110-119 (1997)

${ }^{1}$ http://louisville.edu/speed/bioengineering/faculty/bioengineering-full/dr-aymanel-baz/dr-ayman-el-baz-publications.html 